\title{
Lumbar Disc Degenerative Disease: Disc Degeneration Symptoms and Magnetic Resonance Image Findings
}

\author{
Shafaq Saleem ${ }^{1}$, Hafiz Muhammad Aslam ${ }^{1}$, Muhammad Asim Khan Rehmani ${ }^{2}$, \\ Aisha Raees ${ }^{1}$, Arsalan Ahmad Alvi ${ }^{1}$, Junaid Ashraf ${ }^{3}$ \\ ${ }^{1}$ Neurosurgery Department, Dow Medical College, Dow University of Health Sciences, Karachi, Pakistan \\ ${ }^{2}$ Neurosurgery Department, Civil Hospital, Dow University of Health Sciences, Karachi, Pakistan \\ ${ }^{3}$ Neurosurgery Department, Civil Hospital, Principal Dow Medical College, Dow University of Health Sciences, Karachi, Pakistan
}

\begin{abstract}
Study Design: Cross sectional and observational.
Purpose: To evaluate the different aspects of lumbar disc degenerative disc disease and relate them with magnetic resonance image (MRI) findings and symptoms.

Overview of Literature: Lumbar disc degenerative disease has now been proven as the most common cause of low back pain throughout the world. It may present as disc herniation, lumbar spinal stenosis, facet joint arthropathy or any combination. Presenting symptoms of lumbar disc degeneration are lower back pain and sciatica which may be aggravated by standing, walking, bending, straining and coughing.

Methods: This study was conducted from January 2012 to June 2012. Study was conducted on the diagnosed patients of lumbar disc degeneration. Diagnostic criteria were based upon abnormal findings in MRI. Patients with prior back surgery, spine fractures, sacroiliac arthritis, metabolic bone disease, spinal infection, rheumatoid arthritis, active malignancy, and pregnancy were excluded.

Results: During the targeted months, 163 patients of lumbar disc degeneration with mean age of $43.92 \pm 11.76$ years, came into Neurosurgery department. Disc degeneration was most commonly present at the level of $L 4 / L 5105$ (64.4\%).Commonest types of disc degeneration were disc herniation 109 (66.9\%) and lumbar spinal stenosis 37 (22.7\%). Spondylolisthesis was commonly present at L5/S1 10 (6.1\%) and associated mostly with lumbar spinal stenosis 7 (18.9\%).

Conclusions: Results reported the frequent occurrence of lumbar disc degenerative disease in advance age. Research efforts should endeavor to reduce risk factors and improve the quality of life.
\end{abstract}

Keywords: Claudication; Low back pain; Spondylolisthesis

\section{Introduction}

Lumbar disc degenerative disease is the most common cause of Low back pain throughout the world [1-3]. In the industrialized part of the world low back pain is extremely common. It is the single most common cause of disability at age above 45 years and second most common reason for primary care physician visit [4-6]. People

Received Sep 20, 2012; Revised Nov 20, 2012; Accepted Dec 4, 2012

Corresponding author: Hafiz Muhammad Aslam

Neurosurgery Department, Dow Medical College, Dow University of Health Sciences,

Flat \# 14, 3rd floor, Rafiq Mansion, Cambell road, Off Arambagh, Kararchi, Pakistan

Tel: +92-0345-2460930, E-mail: coolaslam8@hotmail.com 
throughout the world spend more than 100 billion US dollars/year for the treatment of low back pain [2]. Despite the high prevalence of low back pain in both developed and developing nations, it is still enigmatic in terms of cause, diagnosis and treatment [1].

Intervertebral disc is the largest avascular tissue in the body [2], and consists of inner nucleus pulposus, outer annulus fibrosus and cartilage located superiorly and inferiorly. Intervertebral disc resists compression because of the osmotic properties of the proteoglycans. The ability of the disc to resist anterior and lateral shears along with compression and flexion makes the intervertebral disc the most important load bearing component of the spine, beside the facets [5]. Due to loading there is a deformation of the endplate which results in reduced intradiscal pressure, loss of height and adding stress to the surrounding annulus and facet joints. Signs of degeneration includes one or all of the following: diminished disc height, narrowing of facet, spondylophytes and sclerosis of upper and lower endplates, stenosis of spinal canal, narrowing of lateral recess, real or apparent desiccation, fibrosis, diffuse bulging of the annulus beyond the disc space, extensive fissuring (i.e., numerous annular tears), mucinous degeneration of the annulus, defects and sclerosis of the endplates, and osteophytes at the vertebral apophyses [7]. Lumbar degeneration can occur at any level but mainly it occurs on L3-L4 and L4-S1 vertebrae [8-10].

Lumbar disc degenerative disease may present as disc herniation, lumbar spinal stenosis, facet joint arthropathy or their combination. Herniation occurs when nuclear materials protrude or extrude into the perineural space through radial tears of the annulus $[2,7,11]$. Lumbar spinal stenosis is defined as any type of narrowing of spinal canal, nerve root canal or intervertebral foramina. With disc degeneration and loss of disc space height, there are increased stresses on the facet joints with craniocaudal subluxation resulting in arthrosis and osteophytosis, and this condition is termed facet joint arthropathy [7].

The most common symptom associated with lumbar disc degeneration is low back pain and it is due to the presence of neural tissue around the intervertebral disc. The main symptom of disc degeneration after low back pain is sciatica. Features suggestive of sciatica are unilateral or bilateral leg pain radiating to the feet and toes, numbness in dermatomes distribution and positive straight leg raising test. Sciatic pain aggravates on standing, walking, bending, straining and coughing $[9,10]$.
Other symptoms of lumbar disc degeneration are sensory disturbances in legs, claudication, relief of pain when bending forward and weakness [7].

Risk factors for causing lumbar disc degenerative disease include advancing age, socioeconomic status [12], torsional stress [13], smoking, obesity $[11,14,15]$, heavy lifting, vibration [11], trauma, immobilization [5], psychosocial factors, gender, height, hereditary, genetic factors $[12,14]$, occupations like machine drivers, carpenters and office workers [16-18]. Main diagnostic tool and imaging technique for the evaluation of disc degeneration is magnetic resonance imaging (MRI) [19,20].

The basic purpose of conducting this study is to evaluate the relation between different aspects of lumbar degenerative disc disease and their MRI findings; we also assess the relation between lumbar degenerative diseases with symptomatology.

\section{Materials and Methods}

This was a cross sectional and observational study conducted in the general ward and outpatient department of Neurosurgery in Civil Hospital Karachi. The duration of the study was 6 months from January 2012 to June 2012 . A total of 163 diagnosed patients of lumbar disc degenerative diseases were included in this study after obtaining verbal consent. Patients with cervical and thoracic disc degeneration, prior back surgery, spine fractures, sacroiliac arthritis, metabolic bone disease, spinal infection, rheumatoid arthritis, active malignancy, pregnancy and patients having age $<20$ and $>60$ years were excluded. Once the subject was entered in the study, multiplanar MRI was done from the first lumbar to the first sacral vertebra with a 1.5 -tesla imaging system. MRI images were independently evaluated by two neurosurgeons with consensus, one with more than 6 years of experience and a special interest in spine surgery and one with more than 22 years of experience in spine surgery. Each level from L1-S1 was assessed for disc degeneration, using the latest international nomenclature for describing disc pathology. The signal intensity changes of the disc in sagittal sections on $\mathrm{T} 2$-weighted images was graded using a scale from 0 to 3 where $0=$ homogeneous hyper-intense (white), 1=hyper-intense with visible intranuclear cleft (white with a dark band in the equator plane of the disc), $2=$ intermediate signal intensity (all colors between white and black), and 3=hypo-intense (dark disc without vis- 
ible nuclear complex). Changes in the disc contour were described on a nominal scale: $0=$ normal, $1=$ bulge, $2=$ focal protrusion, $3=$ broad based protrusion, $4=$ extrusion, and $5=$ sequestration. Defects in end-plates were graded: $0=$ normal endplates, $1=$ defects and $2=$ large defects. Lumbar disc degeneration was diagnosed if there was either a signal intensity change (grade 2 or 3 ) or a change in disc contour (grade 2 or higher) at one or more lumbar levels. Those with normal signal intensity (grade 0 and 1); normal disc contour (grade 0 or 1), no annular tears, normal endplates and no other pathology in MRI were classified as subjects without disc degeneration $[21,22]$. Spondylolisthesis was measured and diagnosed by the method of Meyerding [23]. The anteroposterior (AP) diameter of the superior surface of the lower vertebral body is divided into quarters and a grade of I-IV is assigned to slips of one, two, three or four quarters of the superior vertebra, but we could not divide our data according to the grades of spondylolisthesis; we simply noted whether spondylolisthesis was present. If not we labeled the patient as free from spondylolisthesis. Patient findings were gathered with a structured questionnaire that included questions regarding biodata (name, age, sex, gender, and date), symptomatology, and status of smoking and MRI findings. The study was ethically approved by Institutional Review Board of Dow Medical College (DUHS). The sample size was calculated using Open-EPI sample size calculator with $p=12 \%, \mathrm{~d}=5 \%$, and $95 \%$ confidence interval. All the data was entered and analyzed through SPSS ver. 19 (IBM Co., Somers, NY, USA). Means and standard deviations were used for continuous data, while frequency and percentages were calculated for categorical data. Correlation of aspects of degeneration with MRI findings and symptomatology were explored by using Spearman rank correlation. All aspects of degeneration were considered individually as $100 \%$ and the percentage in each column add up to $100 \%$. Percentages in the Tables 1-3, which represent the overall result of lumbar degeneration, were evaluated by dividing each variable with 163. Tables 4-6, represent aspects of degeneration: percentages were calculated by dividing each variable with the number of patients of each aspect. Number of effected vertebral levels can be single or multiple and not correlated with sample size; they can be either more than the sample size or less because they can affect a single or multiple levels in the same patient.
Table 1. Table represents the frequency and percentage of LBP aspects of degeneration and MRI findings

\begin{tabular}{|c|c|c|}
\hline S. no & Variable & Frequency (\%) \\
\hline 1 & Low back pain & $163(100.0)$ \\
\hline$A$ & Continous & $90(55.2)$ \\
\hline B & Intermittent & $73(44.8)$ \\
\hline 2 & \multicolumn{2}{|l|}{ Aggravation of pain } \\
\hline$A$ & Walking & $98(60.1)$ \\
\hline B & Standing & $71(43.6)$ \\
\hline C & Lifting & $31(19)$ \\
\hline $\mathrm{D}$ & Sitting & $87(53.4)$ \\
\hline E & Driving & $12(7.4)$ \\
\hline $\mathrm{F}$ & Bending & $81(49.7)$ \\
\hline G & Claudication & $46(28.2)$ \\
\hline \multicolumn{3}{|c|}{ Aspect of degeneration } \\
\hline A & Disc herniation & 109 (66.9) \\
\hline B & Lumbar stenosis & $37(22.7)$ \\
\hline C & Facet joint arthropathy & $4(2.5)$ \\
\hline$D$ & Disc herniation+arthropathy & $4(2.5)$ \\
\hline E & Lumbar stenosis+arthropathy & $2(1.2)$ \\
\hline $\mathrm{F}$ & Disc herniation+ lumbar stenosis & $7(4.3)$ \\
\hline \multicolumn{3}{|c|}{ MRI findings } \\
\hline 1 & Height of disc space reduce & $157(96.3)$ \\
\hline A & $\mathrm{L} 1 / \mathrm{L} 2$ & $28(17.2)$ \\
\hline B & $\mathrm{L} 2 / \mathrm{L} 3$ & $42(25.8)$ \\
\hline C & $\mathrm{L} 3 / \mathrm{L} 4$ & $63(38.7)$ \\
\hline D & $\mathrm{L} 4 / \mathrm{L} 5$ & $120(73.6)$ \\
\hline E & L5/S1 & $96(58.9)$ \\
\hline 2 & Bulge & $115(70.6)$ \\
\hline A & L1/L2 & $8(4.9)$ \\
\hline B & $\mathrm{L} 2 / \mathrm{L} 3$ & $24(14.7)$ \\
\hline C & L3/L4 & $26(16)$ \\
\hline D & L4/L5 & $69(42.3)$ \\
\hline$E$ & L5/S1 & $48(29.4)$ \\
\hline 3 & Protrusion & $104(63.8)$ \\
\hline A & $\mathrm{L} 1 / \mathrm{L} 2$ & $5(3.1)$ \\
\hline B & $\mathrm{L} 2 / \mathrm{L} 3$ & $8(4.9)$ \\
\hline C & L3/L4 & $25(15.3)$ \\
\hline D & L4/L5 & $62(38.0)$ \\
\hline$E$ & L5/S1 & $44(27.0)$ \\
\hline
\end{tabular}

Total number of patients were 163 while nubmer of vertebral levels were not consistent with number of patients because defects are either on single or multiple level. Each variable like low back pain, walking, standing, lifting, driving, sitting, bending, claudication, aspects of degeneration and variable of each magnetic resonance imaging (MRI) findings were consider as $100 \%$. 
Table 2. Table represents the different variables of MRI findings of disc degeneration

\begin{tabular}{|c|c|c|}
\hline S. no & Variable & Frequency (\%) \\
\hline 4 & Extrusion & $27(16.6)$ \\
\hline$A$ & $\mathrm{~L} 1 / \mathrm{L} 2$ & 0 \\
\hline B & L2/L3 & $4(2.5)$ \\
\hline C & $\mathrm{L} 3 / \mathrm{L} 4$ & $2(1.2)$ \\
\hline D & L4/L5 & $8(4.9)$ \\
\hline$E$ & $L 5 / S 1$ & $16(9.8)$ \\
\hline 5 & Disc dessication & $163(100)$ \\
\hline A & $\mathrm{L} 1 / \mathrm{L} 2$ & $29(17.8)$ \\
\hline B & L2/L3 & $40(24.5)$ \\
\hline C & L3/L4 & $71(43.6)$ \\
\hline D & L4/L5 & $123(75.5)$ \\
\hline$E$ & $L 5 / S 1$ & $101(62)$ \\
\hline 6 & Neural canal narrowing & $113(69.3)$ \\
\hline$A$ & $\mathrm{~L} 1 / \mathrm{L} 2$ & $7(4.3)$ \\
\hline B & L2/L3 & $17(10.4)$ \\
\hline C & L3/L4 & $34(20.9)$ \\
\hline D & L4/L5 & $70(42.9)$ \\
\hline $\mathrm{E}$ & $L 5 / S 1$ & $51(31.3)$ \\
\hline 7 & Foraminal narrowing & $137(84.0)$ \\
\hline A & L1/L2 & $7(4.3)$ \\
\hline B & L2/L3 & $21(12.88)$ \\
\hline C & $\mathrm{L} 3 / 14$ & $42(25.8)$ \\
\hline D & L4/L5 & $85(52.1)$ \\
\hline$E$ & $L 5 / S 1$ & $61(37.4)$ \\
\hline 8 & Narrowing of lateral racess & $135(82.8)$ \\
\hline$A$ & $\mathrm{~L} 1 / \mathrm{L} 2$ & $5(3.1)$ \\
\hline B & L2/L3 & $19(11.7)$ \\
\hline C & $\mathrm{L} 3 / \mathrm{L} 4$ & $36(22.1)$ \\
\hline D & L4/L5 & $87(53.4)$ \\
\hline E & $L 5 / S 1$ & $58(35.6)$ \\
\hline 9 & Loss of lumbar lordosis & $50(92)$ \\
\hline 10 & Ligamentum flavum hypertrophy & $62(38)$ \\
\hline A & L11/L2 & $4(2.5)$ \\
\hline B & L2/L3 & $18(11)$ \\
\hline C & $\mathrm{L} 3 / \mathrm{L} 4$ & $27(16.6)$ \\
\hline D & L4/L5 & $46(28.2)$ \\
\hline E & $L 5 / S 1$ & $32(19.6)$ \\
\hline 10 & Facet involment & $90(55.2)$ \\
\hline A & $\mathrm{L} 1 / \mathrm{L} 2$ & $14(8.6)$ \\
\hline B & L2/L3 & $26(16)$ \\
\hline C & L3/L4 & $38(23.3)$ \\
\hline D & L4/L5 & $73(44.8)$ \\
\hline E & $L 5 / S 1$ & $42(25.8)$ \\
\hline
\end{tabular}

Total number of patients were 163 while number of vertebral levels were not consistent with number of patients because defects are either on single or multiple level. Each variable of magnetic resonance imaging (MRI) findings were considered as $100 \%$. Each level of variable were consider as $100 \%$.
Table 3. Table represents the different variables of MRI findings of disc degeneration and sciatica

\begin{tabular}{clc}
\hline S. no & Variable & Frequency (\%) \\
\hline 11 & Spinal nerve involment & $138(84.7)$ \\
\hline A & L1/L2 & $9(5.5)$ \\
\hline B & L2/L3 & $24(14.7)$ \\
\hline C & L3/L4 & $44(27.0)$ \\
\hline D & L4/L5 & $91(55.8)$ \\
\hline E & L5/S1 & $66(40.5)$ \\
\hline 12 & Thecal indentation & $131(80.4)$ \\
\hline A & L1/L2 & $8(4.9)$ \\
\hline B & L2/L3 & $22(13.5)$ \\
\hline C & L3/L4 & $40(24.5)$ \\
\hline D & L4/L5 & $77(47.2)$ \\
\hline E & L5/S1 & $63(38.7)$ \\
\hline 13 & Main vertebrae level & \\
\hline A & L1/L2 & $8(4.9)$ \\
\hline B & L2/L3 & $25(15.3)$ \\
\hline C & L3/L4 & $51(31.3)$ \\
\hline D & L4/L5 & $105(64.4)$ \\
\hline E & L5/S1 & $76(46.6)$ \\
\hline 14 & Spondylolisthesis & $15(9.2)$ \\
\hline A & L1/L2 & 0 \\
\hline B & L2/L3 & $2(1.2)$ \\
\hline C & L3/L4 \\
\hline D & L4/L5 & $2(1.2)$ \\
\hline E & L5/S1 & $5(3.1)$ \\
\hline 15 & Sciatica & $10(6.1)$ \\
\hline A & Right leg & \\
\hline B & Left leg & $43(26.4)$ \\
\hline C & Bilateral leg & $55(33.3)$ \\
\hline D & No radiation & $61(37.4)$ \\
\hline Dta & $4(2.5)$ \\
\hline
\end{tabular}

Total number of patients were 163 while number of vertebral levels were not consistent with number of patients because defects are either on single or multiple level. Each variable of magnetic resonance imaging (MRI) findings and sciatica were considered as 100\%. Each level of variable were consider as $100 \%$.

\section{Results}

There were 163 diagnosed patients with degenerative disease in which 95 (58.3\%) were male and 68 (41.7\%) were females. Patients were between the ages of 20 and 70 years

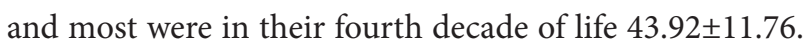
All included patients had history of low back pain, with 
Table 4. Table represents the different aspects of lumbar degeneration and their association with symptomatology and MRI findings

\begin{tabular}{|c|c|c|c|c|c|c|c|c|}
\hline S. no & Variables & $\begin{array}{l}\text { Disc } \\
\text { herniation } \\
(\mathrm{n}=109)\end{array}$ & $\begin{array}{l}\text { Lumbar } \\
\text { stenosis } \\
\text { (n=37) }\end{array}$ & $\begin{array}{l}\text { Facet joint } \\
\text { arthropathy } \\
(n=4)\end{array}$ & $\begin{array}{l}\text { Disc } \\
\text { herniation+facet } \\
\text { joint } \\
\text { arthropatyhy } \\
(\mathrm{n}=4)\end{array}$ & $\begin{array}{l}\text { Lumbar } \\
\text { stenosis+facet } \\
\text { joint } \\
\text { arthropathy } \\
(\mathrm{n}=2)\end{array}$ & $\begin{array}{l}\text { Disc } \\
\text { herniation } \\
\text { +lumbar } \\
\text { astenosis } \\
(\mathrm{n}=7)\end{array}$ & $p$-value \\
\hline 1 & Low back pain & $109(100)$ & 37 (100) & $4(100)$ & $4(100)$ & $2(100)$ & $7(100)$ & \\
\hline 2 & Continous & $63(57.8)$ & $15(40.5)$ & $4(100)$ & $4(100)$ & $2(100)$ & $2(28.6)$ & 0.522 \\
\hline 3 & Intermittent & 46 (42.2) & 22 (59.5) & $0(0)$ & $0(0)$ & $0(0)$ & $5(71.4)$ & 0.522 \\
\hline
\end{tabular}

\begin{tabular}{|c|c|c|c|c|c|c|c|c|}
\hline & $\begin{array}{l}\text { Aggravation of pain } \\
\text { (co-symptom) }\end{array}$ & & & & & & & \\
\hline 4 & Walking & 55 (50.5) & 34 (91.9) & $3(75)$ & $0(0)$ & $0(0)$ & $6(85.7)$ & 0.02 \\
\hline 5 & Standing & $48(\mathbf{4 4 . 0})$ & $20(54.1)$ & $0(0)$ & $0(0)$ & $0(0)$ & $3(42.9)$ & 0.56 \\
\hline 6 & Lifting & $28(25.7)$ & $1(\mathbf{2 . 7})$ & $2(50)$ & $0(0)$ & $0(0)$ & $0(0)$ & 0.03 \\
\hline 7 & Sitting & $65(59.6)$ & 14 (37.8) & $1(25)$ & $3(75)$ & $2(100)$ & $2(28.6)$ & 0.33 \\
\hline 8 & Twisting & $19(17.4)$ & $5(13.5)$ & $0(0)$ & $2(50)$ & $0(0)$ & $2(28.6)$ & 0.94 \\
\hline 9 & Driving & $9(8.3)$ & $1(2.7)$ & $2(50)$ & $0(0)$ & $0(0)$ & $0(0)$ & 0.62 \\
\hline \multirow[t]{2}{*}{10} & Claudication & $0(0)$ & $37(100)$ & $0(0)$ & $0(0)$ & $2(100)$ & $7(100)$ & $<0.01$ \\
\hline & Sciatica & & & & & & & \\
\hline 11 & Right leg & $40(36.7)$ & $3(8.1)$ & $0(0)$ & $0(0)$ & $0(0)$ & $0(0)$ & $<0.01$ \\
\hline 12 & Left leg & 39 (35.8) & $8(21.6)$ & $3(75)$ & $2(50)$ & $2(100)$ & $1(14.3)$ & $<0.01$ \\
\hline 13 & Bilateral leg & $26(\mathbf{2 3 . 9})$ & $26(70.3)$ & $1(25)$ & $2(50)$ & $0(0)$ & $6(85.7)$ & $<0.01$ \\
\hline \multirow[t]{3}{*}{14} & No radiation & $4(3.7)$ & $0(0)$ & $0(0)$ & $0(0)$ & $0(0)$ & $0(0)$ & $<0.01$ \\
\hline & MRI findings & & & & & & & \\
\hline & Height of disc reduce & $103(94.5)$ & $37(100)$ & $4(100)$ & $4(100)$ & $2(100)$ & $7(100)$ & 0.08 \\
\hline$A$ & $\mathrm{~L} 1 / \mathrm{L} 2$ & $9(8.3)$ & $12(32.4)$ & $3(75)$ & $0(0)$ & $0(0)$ & 4 (57.1) & $<0.01$ \\
\hline B & L2/L3 & $11(10.1)$ & $21(56.8)$ & $4(100)$ & $0(0)$ & $2(100)$ & 4 (57.1) & $<0.01$ \\
\hline C & $\mathrm{L} 3 / \mathrm{L} 4$ & $27(24.8)$ & $27(73)$ & $3(75)$ & $0(0)$ & $2(1000$ & $4(57.1)$ & $<0.01$ \\
\hline$D$ & L4/L5 & $73(67.0)$ & 32 (86.5) & $4(100)$ & $2(50)$ & $2(100)$ & $7(100)$ & 0.06 \\
\hline \multirow[t]{2}{*}{$E$} & L5/S1 & $62(56.9)$ & $19(51.4)$ & $4(100)$ & $4(100)$ & $2(100)$ & $5(71.4)$ & 0.24 \\
\hline & BuLGE & 74 (67.9) & $27(73)$ & $2(50)$ & $4(100)$ & $2(100)$ & $6(85.7)$ & 0.23 \\
\hline A & $\mathrm{L} 1 / \mathrm{L} 2$ & $2(1.8)$ & $5(13.5)$ & $1(25)$ & $0(0)$ & $0(0)$ & $0(0)$ & 0.02 \\
\hline B & $\mathrm{L} 2 / \mathrm{L} 3$ & $3(2.8)$ & $13(35.1)$ & $2(50)$ & $0(0)$ & $2(100)$ & $4(57.1)$ & $<0.01$ \\
\hline C & $\mathrm{L} 3 / \mathrm{L} 4$ & $10(9.2)$ & $13(35.1)$ & $1(25)$ & $0(0)$ & $2(100)$ & $0(0)$ & 0.03 \\
\hline$D$ & L4/L5 & $48(44)$ & $15(40.5)$ & $2(50)$ & $2(50)$ & $2(100)$ & $0(0)$ & 0.45 \\
\hline \multirow[t]{2}{*}{$E$} & L5/S1 & $28(25.7)$ & $11(29.7)$ & $1(25)$ & $4(100)$ & $2(100)$ & $2(28.6)$ & 0.07 \\
\hline & PROTRUSION & $73(67)$ & $21(56.8)$ & $1(25)$ & $0(0)$ & $2(100)$ & $7(100)$ & 0.30 \\
\hline A & L1/L2 & $0(0)$ & $5(13.5)$ & $0(0)$ & $0(0)$ & $0(0)$ & $0(0)$ & 0.007 \\
\hline B & $\mathrm{L} 2 / \mathrm{L} 3$ & $1(0.9)$ & $5(13.5)$ & $0(0)$ & $0(0)$ & $2(100)$ & $0(0)$ & 0.001 \\
\hline$C$ & $\mathrm{~L} 3 / \mathrm{L} 4$ & $11(10.1)$ & $12(32.4)$ & $0(0)$ & $0(0)$ & $2(100)$ & $0(0)$ & 0.025 \\
\hline$D$ & L4/L5 & $35(32.1)$ & $17(45.9)$ & $1(25)$ & $0(0)$ & $2(100)$ & $7(100)$ & 0.014 \\
\hline E & L5/S1 & 33 (30.3) & $7(18.9)$ & $0(0)$ & $0(0)$ & $2(100)$ & $2(28.6)$ & 0.23 \\
\hline
\end{tabular}

Each variable like low back pain, walking, standing, lifting, driving, sitting, bending, claudication, aspects of degeneration and variable of each magnetic resonance imaging (MRI) findings were consider as 100\%. Total number of patients of disc herniation were 109, lumbar stenosis were 37,facet joint arthropathy were 4, combination of herniation and arthropathy were 4, comnination of stenosis and arthropathy were 2 and combination of stenosis and herniation were 7, while number of vertebral levels were not consistent with number of patients because defects are either on single or multiple level. Each variable of MRI findings were considered as 100\%. Threshold for significance set at 0.05 significant values were highlighted. 
Table 5. Table represents the different aspects of lumbar degeneration and their association with symptomatology and MRI findings

\begin{tabular}{|c|c|c|c|c|c|c|c|c|}
\hline S.no & Variables & $\begin{array}{l}\text { Disc } \\
\text { herniation } \\
(n=109)\end{array}$ & $\begin{array}{l}\text { Lumbar } \\
\text { stenosis } \\
(n=37)\end{array}$ & $\begin{array}{l}\text { Facet joint } \\
\text { arthropathy } \\
(n=4)\end{array}$ & $\begin{array}{l}\text { Disc } \\
\text { herniation } \\
\text { +facet joint } \\
\text { arthropatyhy } \\
(n=4)\end{array}$ & $\begin{array}{l}\text { Lumbar } \\
\text { stenosis+facet } \\
\text { joint } \\
\text { arthropathy } \\
(\mathrm{n}=2)\end{array}$ & $\begin{array}{l}\text { Disc } \\
\text { herniation } \\
\text { +lumbar } \\
\text { astenosis } \\
(\mathrm{n}=7)\end{array}$ & $p$-value \\
\hline 1 & Extrusion & 21 (19.3) & $5(13.5)$ & $1(25)$ & $0(0)$ & $0(0)$ & $0(0)$ & 0.14 \\
\hline 2 & L1/L2 & $0(0)$ & 0 & 0 & 0 & 0 & 0 & - \\
\hline 3 & L2/L3 & $1(0.9)$ & $3(8.1)$ & 0 & 0 & 0 & 0 & 0.15 \\
\hline 4 & L3/L4 & $1(0.9)$ & $1(2.7)$ & 0 & 0 & 0 & 0 & 0.73 \\
\hline 5 & L4/L5 & $7(6.4)$ & $1(2.7)$ & 0 & 0 & 0 & 0 & 0.18 \\
\hline 6 & L5/S1 & $15(13.8)$ & $0(0)$ & $1(25)$ & 0 & 0 & 0 & 0.02 \\
\hline 7 & DISC dessication & 109 (100) & 37 (100) & $4(100)$ & $4(100)$ & $2(100)$ & $7(100)$ & \\
\hline 8 & $\mathrm{~L} 1 / \mathrm{I} 2$ & $10(9.2)$ & $12(32.4)$ & $3(75)$ & $0(0)$ & $0(0)$ & $4(57.1)$ & $<0.01$ \\
\hline 9 & L2/I3 & $10(9.2)$ & $20(54.1)$ & $3(75)$ & $0(0)$ & $2(100)$ & $5(71.4)$ & $<0.01$ \\
\hline 10 & L3/14 & $33(30.3)$ & $27(73)$ & $3(75)$ & 0 & $2(100)$ & $6(85.7)$ & $<0.01$ \\
\hline 11 & L4/L5 & $78(71.6)$ & $30(81.1)$ & $4(100)$ & $2(50)$ & $2(100)$ & $7(100)$ & 0.08 \\
\hline 12 & $\mathrm{~L} 5 / \mathrm{S} 1$ & $65(59.6)$ & $20(54.1)$ & $4(100)$ & $4(100)$ & $2(100)$ & $6(85.7)$ & 0.17 \\
\hline 13 & Neural canal narrowing & $64(58.7)$ & $37(100)$ & $0(0)$ & $3(75)$ & $2(100)$ & $7(100)$ & $<0.01$ \\
\hline 14 & $\mathrm{~L} 1 / \mathrm{L} 2$ & $0(0)$ & $5(13.5)$ & $0(0)$ & 0 & 0 & 2 (28.6) & $<0.01$ \\
\hline 15 & L2/L3 & $0(0)$ & $13(35.1)$ & 0 & 0 & $2(100)$ & $2(28.6))$ & $<0.01$ \\
\hline 16 & L3/L4 & $7(6.4)$ & $23(62.2)$ & 0 & 0 & $2(100)$ & $2(28.6)$ & $<0.01$ \\
\hline 17 & L4/L5 & $32(29.4)$ & $28(75.7)$ & 0 & $1(25)$ & $2(100)$ & $7(100)$ & $<0.01$ \\
\hline 18 & L5/S1 & $34(31.2)$ & $11(29.7)$ & 0 & $2(50)$ & $2(100)$ & 2 (28.6) & 0.89 \\
\hline 19 & Foraminal narrowing & $86(78.9)$ & $37(100)$ & $2(50)$ & $3(75)$ & $2(100)$ & $7(100)$ & 0.02 \\
\hline 20 & $\mathrm{~L} 1 / \mathrm{L} 2$ & 0 & $5(13.5)$ & 0 & 0 & 0 & $2(28.6)$ & $<0.01$ \\
\hline 21 & L2/L3 & $2(1.8)$ & $15(40.5)$ & 0 & 0 & $2(100)$ & $2(28.6)$ & $<0.01$ \\
\hline 22 & L3/L4 & $12(11)$ & $24(64.9)$ & $2(50)$ & $0(0)$ & $2(100)$ & $2(28.6)$ & $<0.01$ \\
\hline 23 & L4/L5 & $46(42.2)$ & $27(73)$ & $2(50)$ & $1(25)$ & $2(100)$ & $7(100)$ & $<0.01$ \\
\hline 24 & $\mathrm{~L} 5 / \mathrm{S} 1$ & $41(31.6)$ & $10(27)$ & $2(50)$ & $2(50)$ & $2(100)$ & $4(57.1)$ & 0.72 \\
\hline 25 & $\begin{array}{l}\text { Lateral; racess } \\
\text { narrowing }\end{array}$ & $85(78)$ & 36 (97.3) & $2(50)$ & $3(75)$ & $2(100)$ & $7(100)$ & 0.03 \\
\hline 26 & L1/L2 & 0 & $5(13.5)$ & 0 & 0 & 0 & 0 & 0.007 \\
\hline 27 & L2/L3 & $1(0.9)$ & 14 (37.8) & 0 & 0 & $2(100)$ & $2(28.6)$ & $<0.01$ \\
\hline 28 & L3/L4 & $9(8.3)$ & $23(62.2)$ & $2(50)$ & 0 & $2(100)$ & 0 & $<0.01$ \\
\hline 29 & L4/L5 & $48(44)$ & $27(73.6)$ & $2(50)$ & $1(\mathbf{2 5 . 0})$ & $2(100)$ & $7(100)$ & 0.001 \\
\hline 30 & L5/S1 & $40(36.7)$ & $10(27)$ & $2(50)$ & $2(50)$ & $2(100)$ & $2(28.6)$ & 0.858 \\
\hline 31 & Loss of lumbar lordosis & $101(92.7)$ & $32(86.5)$ & $4(100)$ & $4(100)$ & $2(100)$ & $7(100)$ & 0.918 \\
\hline
\end{tabular}

Each variable like low back pain, walking, standing, lifting, driving, sitting, bending, claudication, aspects of degeneration and variable of each magnetic resonance imaging (MRI) findings were consider as 100\%. Total number of patients of disc herniation were 109, lumbar stenosis were 37, facet joint arthropathy were 4, combination of herniation and arthropathy were 4. Comnination of stenosis and arthropathy were 2 and combination of stenosis and herniation were 7, while number of vertebral levels were not consistent with number of patients because defects are either on single or multiple level. Each variable of MRI findings were considered as $100 \%$. Threshold for significance set at 0.05 and significant values were bold. 
Table 6. Table represents the different aspects of lumbar degeneration and their association with symptomatology and MRI findings

\begin{tabular}{|c|c|c|c|c|c|c|c|}
\hline S.no Variables & $\begin{array}{l}\text { Disc } \\
\text { herniation } \\
(n=109)\end{array}$ & $\begin{array}{l}\text { Lumbar } \\
\text { stenosis } \\
(n=37)\end{array}$ & $\begin{array}{l}\text { Facet } \\
\text { joint } \\
\text { arthropathy } \\
(n=4)\end{array}$ & $\begin{array}{l}\text { Disc } \\
\text { herniation } \\
\text { +facet } \\
\text { joint } \\
\text { arthropatyhy } \\
(\mathrm{n}=4)\end{array}$ & $\begin{array}{l}\text { Lumbar } \\
\text { stenosis } \\
+ \text { facet } \\
\text { joint } \\
\text { arthropathy } \\
(\mathrm{n}=2)\end{array}$ & $\begin{array}{l}\text { Disc } \\
\text { herniation } \\
\text { +lumbar } \\
\text { astenosis } \\
(\mathrm{n}=7)\end{array}$ & $p$-value \\
\hline
\end{tabular}

1 Ligamentum flavum hypertrophy

$23(21.1) \quad 28(75.7) \quad 0(0)$

$3(75)$

2 (100)

6 (85.7)

$<0.01$

$2 \quad \mathrm{~L} 1 / \mathrm{L} 2$

$0(0)$

2 (5.4) $0(0)$

0 (0)

$0(0)$

2 (28.6)

0.001

3 L2/L3

2 (1.8)

$12(32.4)$

$0(0)$

$0(0)$

2 (100)

2 (28.6)

$<0.01$

$4 \quad$ L3/L4

4 (3.7)

19 (51.4)

$0(0)$

$0(0)$

2 (100)

2 (28.6)

$<0.01$

$5 \quad$ L/L5

$16(14.7)$

19 (51.4)

$3(75.0)$

$2(100)$

6 (85.7)

$<0.01$

$6 \quad$ L5/S

$11(10.1)$

$11(29.7)$

$0(0)$

$3(75)$

2 (100)

5 (71.4)

$<0.01$

7 Facet involment

41 (37.6)

$35(94.6)$

$3(75)$

2(100)

$6(85.7)$

$<0.01$

$8 \quad$ L1/L2

$9 \quad$ L2/L3

4 (3.7)

$7(18.9)$

1 (25)

$0(0)$

$0(0)$

2 (28.6)

0.02

$10 \quad$ L3/L

11 L4/L5

6 (5.5)

1 (25)

0

2 (100)

2 (28.6)

$<0.01$

$12 \quad$ L5/S1

30 (27.5)

$25(67.6)$

$3(75.0)$

$0(0)$

2 (100)

2 (28.6)

$<0.01$

13 Spinal nerves

$16(14.7)$

29 (78.4)

3 (75.0)

3 (75.0)

2 (100)

$6(85.7)$

$<0.01$

$14 \quad$ L1/L2

89 (81.7)

16 (43.2)

$3(75.0)$

$3(75.0)$

2 (100)

2 (28.6)

$<0.01$

$15 \quad \mathrm{~L} 2 / \mathrm{L} 3$

2 (1.8)

35 (94.6)

2 (50)

$3(75.0)$

2 (100)

$7(100)$

0.176

$16 \quad \mathrm{~L} 3 / \mathrm{L}$

4 (3.7)

7 (18.9)

$0(0)$

$0(0)$

$0(0)$

$0(0)$

0.01

17 L4/L5

14(12.8)

16 (43.2)

0 (0)

$0(0)$

2 (100)

2 (28.6)

$<0.01$

18 L5/S

50 (45.9)

24 (64.9)

2 (50)

$0(0)$

2 (100)

2 (28.6)

$<0.01$

19 Thecal compression

42 (38.5)

29 (78.4)

1 (25)

2 (100)

7 (100)

$<0.01$

$20 \quad \mathrm{~L} 1 / \mathrm{L}$

83(76.1)

$1 4 \longdiv { 3 7 . 8 0 }$

2 (50)

2 (50)

2 (100)

$4(57.1)$

$<0.32$

$21 \quad \mathrm{~L} 2 / \mathrm{L} 3$

$1(0.9)$

37 (100)

1 (25)

1 (25)

$2(100)$

$7(100)$

0.14

$22 \quad \mathrm{~L} 3 / \mathrm{L} 4$

2 (1.8)

$5(13.5)$

$0 \quad 0$

$16(43.2) \quad 0$

$2(100)$

2 (28.6)

0.001

$23 \quad \mathrm{~L} 4 / \mathrm{L}$

12 (11)

$24(64.9)$

0

2 (100)

2 (28.6)

$<0.01$

$24 \quad \mathrm{~L} 5 / \mathrm{S}$

41 (37.6)

28 (75.7)

0

$2(100)$

2 (28.6)

$<0.01$

$25 \quad$ Vertebral level

$26 \quad \mathrm{~L} 1 / \mathrm{L}$

$27 \quad \mathrm{~L} 2 / \mathrm{L} 3$

43 (39.4)

$12(32.4)$

0

1 (25)

2 (100)

5 (71.4)

0.001

2 (100)

5 (71.4)

0.98

$28 \quad L 3 / L$

$29 \quad \mathrm{~L} 4 / \mathrm{L} 5$

2 (1.8)

$5(13.5)$

1 (25)

$5(4.6)$

17 (45.9)

$1(25)$

0

0

$18(16.5) \quad 28(75.7) \quad 3(75)$

$30 \quad$ L5/S1

$61(56)$

$31(83.8) \quad 4(100)$

0

0

$2 \cos 2(115)$

$2(100)$

$0(0)$

$2(100)$

2 (100)

$4(100)$

$2(100)$

$7(6.4)$

$11(29.7) \quad 4(100)$

0

0

$0 \quad 0$

$\begin{array}{lll}32 & \mathrm{~L} 1 / \mathrm{L} 2 & 0 \\ 33 & \mathrm{~L} 2 / \mathrm{L} 3 & 0\end{array}$

0

2 (5.4) 0

0

$2(5.4)$

$1(0.9)$

4 (10.8)

$6(5.5)$

$3(8.1)$

0
0
0

0

0
0
0
0
0

\begin{tabular}{lll}
$2(100)$ & $5(71.4)$ & 0.25 \\
0 & $1(14.3)$ & 0.14 \\
0 & 0 & - \\
0 & 0 & 0.09 \\
0 & 0 & 0.09 \\
0 & 0 & 0.06 \\
0 & $1(14.3)$ & 0.65 \\
\hline
\end{tabular}

Each variable like low back pain, walking, standing, lifting, driving, sitting, bending, claudication, aspects of degeneration and variable of each magnetic resonance imaging (MRI) findings were consider as 100\%. Total number of patients of disc herniation were 109, lumbar stenosis were 37 , facet joint arthropathy were 4, combination of herniation and arthropathy were 4. Comnination of stenosis and arthropathy were 2 and combination of stenosis and herniation were 7, while number of vertebral levels were not consistent with number of patients because defects are either on single or multiple level. Each variable of MRI findings were considered as $100 \%$. Threshold for significance set at 0.05 and significant values were bold. 
$90(55.2 \%)$ patients had continuous type of pain while 73 (44.8\%) had intermittent type of pain (Table 1). Most patients 61 (37.4\%) had bilateral sciatica: 43 (26.4\%) had right side sciatica, left side sciatica was present in 55 (33.3\%), and four (2.5\%) had no sciatica complaint. Neurogenic claudication was positive in $46(28.2 \%)$ patients (Table 3). Aggravation of low back and sciatic pain occurs mostly during walking 98 (60.1\%) while other positions and postures which aggravate the pain were bending 81 (49.7), standing 71 (43.6\%), sitting 87 (53.4\%), lifting 31 (19\%), and driving 12 (7.4\%) (Table 1).

There were six aspects of degeneration evaluated in this study. Disc herniation was the most frequent (109, $66.9 \%$ ), others were lumbar stenosis $(37,22.7 \%)$, facet joint arthropathy (4,2.5\%), combination of disc herniation and facet joint arthropathy (4,2.5\%), combination of lumbar stenosis and facet joint arthropathy $(2,1.2 \%)$ and combination of disc herniation and lumbar stenosis (7, $4.3 \%$ ) patients (Table 1).

The most common levels of disc degeneration were L4/ L5 and L5/S1, seen in 105 (64.4\%) and 76 (46.6\%) patients respectively. The mean age of patients with disc degeneration at levels L3/4 (47.75 \pm 14.13$), \mathrm{L} 2 / 3$ (55.5 \pm 7.39$)$ and L1/2 (50.39 \pm 9.36$)$ was significantly higher than the mean age of patients with disc degeneration at lower levels, L4/5 (45.34 \pm 11.48$)$ and L5/S1 (43.14 \pm 12.18$)$.

After thorough MRI study it was concluded that the space between 2 vertebrae was reduced in 157 (96.3\%) patients and it was most commonly reduced at the level of L4/L5 120 (73.6\%) and L5/S1 96 (58.9\%) while bulge of disc was present in 115 (70.6\%) patients, also most commonly occurring at L4/L5 69 (42.3\%) and L5/S1 48 (29.4\%). Protrusion of intervertebral disc was found in $104(63.8 \%)$ patients; occurring most commonly at L4/L5 62 (38\%) and L5/S1 44 (27\%) level (Table 1). Extrusion of disc was present in 27 (16.7\%) patients, most often at L5/ S1 16 (9.8\%) and L4/L5 8(4.9\%) followed by four (2.5\%) at L2/L3 and two (1.2\%) at L3/L4 (Table 2). Disc desiccation was present in all patients $163(100 \%)$, primarily at the level of L4/L5 123 (75.5\%) and L5/S1 101 (62\%). Neural canal narrowing was noted in $113(69.3 \%)$ patients, most often at the level of L4/L5 70 (42.9\%) and L5/S1 51 (31.3\%). Foraminal narrowing was present in $137(84 \%)$ patients, mainly at the level of L4/L5 85 (52.1\%) and L5/ S1 61 (37.4\%). Narrowing of lateral recess occurred in $135(82.8 \%)$ patients, primarily at the level of L4/L5 87 (53.4\%) and L5/S1 58 (35.6\%). Ligamentum flavum hy- pertrophy occurred in $62(38 \%)$ patients most commonly at the level of L4/L5 46 (28.2\%) and L5/S1 32 (19.6\%). During the degeneration process a facet was involved in $90(55.2 \%)$ patients largely seen at the level of L4/L5 73 (44.8\%) and L5/S1 42 (25.8\%) (Table 2). Spinal nerve was pressing in 138 (84.7\%) patients, a common occurrence at the level of L4/L5 91 (55.8\%) and L5/S1 66 (40.5\%).

Prevalence of spondylolisthesis was 15 (9.2\%) mostly seen at the level of L5/S1 10 (6.1\%) (Table 3).

The mean age of patients with lumbar disc herniation was $39.92 \pm 10.92$ years and the most common level of disc herniation were L4/L5 (61, 56\%) $(p=0.02)$ and L5/S1 (55, $50.5 \%)(p=0.25)$ patients (Table 3$)$.

MRI study concluded that the space between 2 vertebrae was reduced in 103 (94.5\%) ( $p=0.08)$ patients and it was mainly reduced at the level of L4/L5 73 (67.0\%) $(p=0.06)$ and L5/S1 $62(56.9 \%)(p=0.24)$ while a bulging of a disc was present in $74(67.90 \%)(p=0.23)$ patients, a common occurrence at L4/L5 $48(44 \%)(p=0.45)$ and L5/S1 28 (25.7\%) $(p=0.07)$. Protrusion of an intervertebral disc was found in $73(67 \%)(p=0.30)$ patients, most often at L4/L5 35 (32.1\%) ( $p=0.01)$ and L5/S1 33 (30\%) (0.23) levels (Table 1). Extrusion of disc was present in $21(19.3 \%)(p=0.14)$ patients, most commonly at L5/S1 $15(13.8 \%)(p=0.02)$ and L4/L5 7 (6.4\%) $(p=0.18)$. Disc desiccation was present in all patients $109(100 \%)$ at the level of L4/L5 78 (71.6\%) ( $p=0.08$ ) and L5/S1 65 (59.6\%) $(p=0.17)$. Neural canal narrowing was noted in $64(58.7 \%)$ $(p \leq 0.01)$ patients and it was commonly present at the level of L4/L5 $32(29.47 \%)(p<0.01)$ and L5/S1 $34(31.2 \%)$ $(p=0.89)$. Foraminal narrowing was present in $86(78.9 \%)$ $(p=0.02)$ patients, primarily at L4/L5 $46(42.2 \%)(p \leq 0.01)$ and L5/S1 41 (31.6\%) $(p=0.72)$.Narrowing of lateral recess was found to occur at $85(78 \%)(p=0.03)$ patients and it was mostly found at the level of L4/L5 48 (44\%) $(p=0.001)$ and L5/S1 40 (36.7\%) ( $p=0.858)$. Loss of lumbar lordosis was present in $101(92.7 \%)(p=0.91)$ (Table 2 ) and ligamentum flavum hypertrophy occurred in 23 $(21.1 \%)(p<0.01)$ patients, a common occurrence at the level of L4/L5 $16(14.7 \%)(p \leq 0.01)$ and L5/S1 $11(10.1 \%)$ $(<0.01)$. During the degeneration process a facet was involved in $41(37.6 \%)(p<0.01)$ patients, commonly at L4/ L5 $30(27.5 \%)(p<0.01)$ and L5/S1 $16(14.7 \%)(p<0.01)$. A spinal nerve was pressing in $89(81.7 \%)(p=0.176)$ patients, usually at L4/L5 $50(45.9 \%)(p<0.01)$ and L5/S1 42 $(38.5 \%)(p<0.32)$. Thecal indentation was present in 83 (76.1\%) ( $p=0.14)$ patients, which mostly occurred at the 
level of L4/L5 41 (37.6\%) ( $p=0.001)$ and L5/S1 43 (39.4\%) $(p=0.98)$.

The prevalence of Spondylolisthesis was seven (6.4\%) $(p=0.14)$ and it commonly occurred at L5/S1 $6(5.5 \%)$ $(p=0.65)$ (Table 3).

The mean age of patients with lumbar stenosis was $51.91 \pm 10.92$ year old. The most common level of lumbar spinal stenosis was L4/L5 $4(100 \%)(p=0.02)$ patients (Table 3).

MRI study also showed that the space between 2 vertebrae was reduced in $37(100 \%)(p=0.08)$ patients, primarily at the level of L4/L5 $32(86.5 \%)(p=0.086)$ and L5/S1 $19(51.4 \%)(p=0.24)$ while a bulge of disc was present in $27(73 \%)(p=0.23)$ patients, a common occurrence at L4/L5 $15(40.5 \%)(p=0.45)$ and L5/S1 $11(29.7 \%)$ $(p=0.07)$. Protrusion of intervertebral disc was seen in 21 (56.8\%) ( $p=0.30)$ patients, commonly occurring at L4/ L5 $17(45.9 \%)(p=0.01)$ (Table 1). Extrusion of disc was present in five $(13.5 \%)(p=0.14)$ patients mainly at L2/ L3 $3(8.1 \%)(p=0.15)$. Disc desiccation was present in all patients $109(100 \%)$ and it was present mostly at the level of L4/L5 30 (81.1\%) ( $p=0.08)$. Neural canal narrowing was observed in $37(100 \%)(p \leq 0.01)$ patients commonly at L4/L5 $28(75.7 \%)(p<0.01)$. Foraminal narrowing was present in $37(100 \%)(p=0.02)$ patients mostly at L4/L5 $27(73 \%)(p \leq 0.01)$. Narrowing of lateral recess occurred in $36(97.3 \%)(p=0.03)$ patients, mostly found at the level of L4/L5 27 (73.6\%) ( $p=0.001)$. Loss of lumbar lordosis present in $32(86.5 \%)(p=0.91)$ (Table 2). Ligamentum flavum hypertrophy occurred in $28(75.7 \%)(p<0.01)$ patients, mostly at L4/L5 19 (51.4\%) ( $p \leq 0.01)$. During degeneration process facets were also involved in $35(94.6 \%)$ $(p<0.01)$ patients mostly at L4/L5 $29(78.4 \%)(p<0.01)$ and spinal nerve was compressed in $35(94.6 \%)(p=0.17)$ patients mainly at L4/L5 $29(78.4 \%)(p<0.01)$. Thecal indentation was evident in 37 (100\%) patients, occurring mostly at L4/L5 28 (75.7\%) $(p=0.001)$

The prevalence of spondylolisthesis was seven (18.9\%) $(p=0.14)$ and it was mostly occurring at the level of L5/S1 3 (8.1\%) (Table 3).

The mean age of patients with facet joint arthropathy was $57.50 \pm 5.19$ and commonly present at the level of L4/ L5 and L5/S1, which was found in four $(100 \%)(p=0.02)$ and $4(100 \%)(p=0.25)$ patients (Table 3$)$.

Spondylolisthesis, neural canal narrowing and ligamentum flavum hypertrophy was not present in facet joint arthropathy (Table 3).
Rest of the comparison, correlations and results were given in tables.

\section{Discussion}

With increasing longevity of life and aging populations, the prevalence and associated clinical disability related to disc degeneration disease is on the rise. Lumbar disc degeneration was the most common cause of low back pain around the globe and it was observed that major aspect of disc degeneration was disc herniation. Modern development of MRI scans allows an excellent noninvasive means of imaging the entire lumbar spine. Its contrast, sensitivity and multi planer images clarify the disc anatomy within or adjacent spine.

Gender comparison revealed that significantly men were more prone to suffered from degeneration, an alarming problem for society. The findings of our study was consistent with other studies [24,25]. This result reinforces the general perception that men are more susceptible to disc degeneration than women, most likely due to increased mechanical stress and injury [26].

Most cases of disc degeneration were observed in 4 th and 5th decade of life in our study, comparable with other studies $[27,28]$. It was also revealed that in older patients degeneration more often had higher level lumbar disc degeneration (L1-L2, L2-L3, L3-L4) whereas young age people had disc degeneration at lower levels (L4-5, L5S1) $[24,27]$.

Aspects of degeneration that were evaluated in our study were disc degeneration, lumbar spinal stenosis, facet joint arthropathy and their combination. Disc herniation was more common among others as indicated in another study [29].

In the current case series MR imaging study, we investigated the prevalence of lumbar disc abnormality in a series of 20- to 70-year-old patients with back pain. It was found that disc degeneration with diffuse disc changes in the lower lumbar spine was more commonly found at L4L5 and L5-S1 which had the highest rate of degeneration and L1-L2 had the lowest $[1,29,30]$. This caudo-cranial direction pattern was also followed by disc herniation. These findings support the fact that mechanical characteristics of the discs are greater in those that are adjacent to fused lumbar vertebrae, favoring degeneration, with high disc involvement observed more commonly at lower lumbar level. It was evaluated and confirmed that with 
aging the loss of proteoglycans from the lumbosacral disc exceeds that from the lower lumbar discs because of its proximity to a rigid segment, that is, the sacrum. This relative fixation and associated facet arthropathy results in greater stresses at the more rostral angles, leading to disc herniation moving cranially, a mirror spread of disc degeneration [27]. The prevalence of disc herniation in diagnosed patients of degeneration was more common than any other aspects and we found that prevalence of L4-L5 was much higher than L1-L2, L2-L3, L3-L4, and L5-S1. It also can be deduced that the lower the lumbar level the higher the prevalence of disc herniation $[1,30]$.

Very few patients had the disability on a single level, and most patients had degeneration on multiple levels; these findings were also consistent with past studies [29].

It was observed in our study that most patients had continuous low back pain, in contrast to the previous study [31]. Along with low back pain, sciatica was also one of the main complaints [32]. Most patients were complaining of unilateral sciatic pain, as shown in a study conducted in Pakistan [10]. It was also confirmed from our study that after low back pain and sciatica, the main complaint of patients with disc degeneration was neurogenic claudication [32], a main feature of lumbar spinal stenosis. It was $100 \%$ positive in all cases of "Lumbar stenosis" and combination of "stenosis and herniation." Low back pain and sciatica were more aggravated during walking and standing position, agreeing with other studies [33-36]. These findings signify the fact that during walking and standing there was decompensation of vascular flow to the spinal nerves, resulting in arterial ischemia as well as venous congestion [33].

It was also concluded that the lower back pain and sciatica were due to nerve root compression, which was significantly associated with disc degeneration [37]. Interestingly there were also $15.7 \%$ of patients who had no visible nerve compression in MR imaging, reflecting the disc impairment without nerve compression as in another study [38].

Disc herniation had the features of disc protrusion, extrusion and disc space narrowing. There was a significant association between disc protrusion and lumbar disc herniation but disc space narrowing was not significantly associated with it [30].

With the beginning of degeneration there was a greater loss of water from the nucleus pulposus resulting in the loss of hydrostatic properties of the disc with an overall reduction of hydration to about $70 \%$, with loss of water, nucleus pulposus become desiccated and it was also shown in our study that all aspects had their disc in desiccated form either at single or multiple levels as shown in a past study [7].

Spinal lumbar stenosis can involve the central canal, lateral recess, foramina or combination of these. All of them start to stenosed when there is narrowing of disc space with or without disc bulging and ligamentum flavum hypertrophy. It was concluded that all MRI of stenosis patients had reduced disc space but not all had bulging and ligamentum flavum hypertrophy as seen in a past study [39]. It was also found that protrusion and facet involvement was present in nearly all cases of stenosis. Our findings confirm the pathways indicated in past studies regarding lumbar stenosis, which consist of reduced disc space height, then protrusion, ligamentum flavum hypertrophy, and last, facet involvement [33]. Further results of our study are that nearly all patients had the combination of central, formanal and lateral recess stenosis in contrast to another study conducted on similar topic [40].

Ligamentum flavum hypertrophy can reduce the diameter of the spinal canal posteriorly and it was significantly more associated with lumbar stenosis than others. It was positively associated with L2-L3, L3-L4, and L4-L5 as shown in a previous study conducted in Turkey [41]. In our study hypertrophy of ligamentum flavum was also significantly associated with disc herniation on all levels except L1-L2, same as signify in previous study [41].

Spondylolisthesis was more commonly found in the patients of lumbar stenosis as compared to disc herniation, reflecting the fact that during stenosis, laxity of capsules and ligaments may result in the development of spondylolisthesis [33]. Spondylolisthesis was mainly present at L4-L5 same as seen in a previous study [7]. The presence of degenerative listhesis at that level was thought to be related to the more sagittal orientation of facet joint that makes them increasingly prone to anterior displacement [7].

We established the baseline data for longitudinal study prospective study and the current study also adds information to the international research literature. This study sees the spine in different aspects and can serve as a reason to improve diagnostic technique in this field of spine. In this we have taken detailed histories which will help the medical profession to understand better spinal degeneration symptomatology. The limitation of our study was 
that it does not discuss risk factors but this can serve as motive to conduct further research. It was also surprising that research on spine and lumbar degeneration was scarce in the hugely populated country of Pakistan. This study was one of the pioneer researches in the field of spine in Pakistan and it will serve as a guide for future researchers.

\section{Conclusions}

This work may lead to new and exciting advances in the field of spine research. The lumbar discs most often affected by degeneration that leads to herniation and stenosis are L4-5 and L5-S1, most probably because of a combination of longstanding degeneration and subsequent change in the ability of the disc to resist applied stress. Awareness is needed of this very common problem and proper protective measures can be taken to prevent the disease in early age, like giving proper time for exercise and prolonging life. It is very necessary to keep risk factors like smoking, weight lifting, prolonged driving, psychosocial factors, and accidents away from our life style.

\section{Conflict of Interest}

No potential conflict of interest relevant to this article was reported.

\section{Acknowledgments}

We acknowledge all the staff members of the Neurosurgery Department, Dow Medical College, Dow University of Health Sciences, who assisted us in completion of this project.

\section{References}

1. Weiler C, Lopez-Ramos M, Mayer HM, et al. Histological analysis of surgical lumbar intervertebral disc tissue provides evidence for an association between disc degeneration and increased body mass index. BMC Res Notes 2011;4:497.

2. Choi YS. Pathophysiology of degenerative disc disease. Asian Spine J 2009;3:39-44.

3. Quint U, Wilke HJ. Grading of degenerative disk disease and functional impairment: imaging versus patho-anatomical findings. Eur Spine J 2008;17:1705-
13.

4. Hicks GE, Morone N, Weiner DK. Degenerative lumbar disc and facet disease in older adults: prevalence and clinical correlates. Spine (Phila Pa 1976) 2009;34:1301-6.

5. Palepu V, Kodigudla M, Goel VK. Biomechanics of disc degeneration. Adv Orthop 2012;2012:726210.

6. Lehtola V, Luomajoki H, Leinonen V, Gibbons S, Airaksinen $\mathrm{O}$. Efficacy of movement control exercises versus general exercises on recurrent sub-acute nonspecific low back pain in a sub-group of patients with movement control dysfunction. Protocol of a randomized controlled trial. BMC Musculoskelet Disord 2012;13:55.

7. Modic MT, Ross JS. Lumbar degenerative disk disease. Radiology 2007;245:43-61.

8. David G, Ciurea AV, Iencean SM, Mohan A. Angiogenesis in the degeneration of the lumbar intervertebral disc. J Med Life 2010;3:154-61.

9. Neuropathy-sciatic nerve; sciatic nerve dysfunction; low back pain-sciatica [Internet]. Bethesda (MD): A.D.A.M. Inc.; c2013 [cited 2012 Aug 12]. Available from: http://www.ncbi.nlm.nih.gov/pubmedhealth/ PMH0001706/.

10. Bakhsh A. Long-term outcome of lumbar disc surgery: an experience from Pakistan. J Neurosurg Spine 2010;12:666-70.

11. Baldwin NG. Lumbar disc disease: the natural history. Neurosurg Focus 2002;13:E2.

12. Katz JN. Lumbar disc disorders and low-back pain: socioeconomic factors and consequences. J Bone Joint Surg Am 2006;88 Suppl 2:21-4.

13. Ala-Kokko L. Genetic risk factors for lumbar disc disease. Ann Med 2002;34:42-7.

14. Kanayama M, Togawa D, Takahashi C, Terai T, Hashimoto T. Cross-sectional magnetic resonance imaging study of lumbar disc degeneration in 200 healthy individuals. J Neurosurg Spine 2009;11:5017.

15. Liuke M, Solovieva S, Lamminen A, et al. Disc degeneration of the lumbar spine in relation to overweight. Int J Obes (Lond) 2005;29:903-8.

16. Battie MC, Videman T, Gibbons LE, Fisher LD, Manninen H, Gill K. 1995 Volvo Award in clinical sciences. Determinants of lumbar disc degeneration. A study relating lifetime exposures and magnetic resonance imaging findings in identical twins. Spine 
(Phila Pa 1976) 1995;20:2601-12.

17. Luoma K, Vehmas T, Riihimaki H, Raininko R. Disc height and signal intensity of the nucleus pulposus on magnetic resonance imaging as indicators of lumbar disc degeneration. Spine (Phila $\mathrm{Pa} 1976$ ) 2001;26:680-6.

18. Battie MC, Videman T, Gibbons LE, et al. Occupational driving and lumbar disc degeneration: a casecontrol study. Lancet 2002;360:1369-74.

19. Taher F, Essig D, Lebl DR, et al. Lumbar degenerative disc disease: current and future concepts of diagnosis and management. Adv Orthop 2012;2012:970752.

20. Tertti M, Paajanen H, Laato M, Aho H, Komu M, Kormano M. Disc degeneration in magnetic resonance imaging. A comparative biochemical, histologic, and radiologic study in cadaver spines. Spine (Phila $\mathrm{Pa}$ 1976) 1991;16:629-34.

21. Fardon DF, Milette PC; Combined Task Forces of the North American Spine Society, American Society of Spine Radiology, and American Society of Neuroradiology. Nomenclature and classification of lumbar disc pathology. Recommendations of the Combined task Forces of the North American Spine Society, American Society of Spine Radiology, and American Society of Neuroradiology. Spine (Phila Pa 1976) 2001;26:E93-E113.

22. Eskola PJ, Kjaer P, Daavittila IM, et al. Genetic risk factors of disc degeneration among 12-14-year-old Danish children: a population study. Int J Mol Epidemiol Genet 2010;1:158-65.

23. Kalichman L, Hunter DJ. Diagnosis and conservative management of degenerative lumbar spondylolisthesis. Eur Spine J 2008;17:327-35.

24. Miller JA, Schmatz C, Schultz AB. Lumbar disc degeneration: correlation with age, sex, and spine level in 600 autopsy specimens. Spine (Phila Pa 1976) 1988;13:173-8.

25. de Schepper EI, Damen J, van Meurs JB, et al. The association between lumbar disc degeneration and low back pain: the influence of age, gender, and individual radiographic features. Spine (Phila Pa 1976) 2010;35:531-6.

26. Wang YX, Griffith JF. Effect of menopause on lumbar disk degeneration: potential etiology. Radiology 2010;257:318-20.

27. Skaf GS, Ayoub CM, Domloj NT, Turbay MJ, ElZein C, Hourani MH. Effect of age and lordotic angle on the level of lumbar disc herniation. Adv Orthop 2011;2011:950576.

28. Karunanayake A, Pathmeswaran A, Wijayaratne LS. Radiological changes of the spine in patients with chronic low backache. Med Today 2007;5:6-9.

29. Boden SD, Davis DO, Dina TS, Patronas NJ, Wiesel SW. Abnormal magnetic-resonance scans of the lumbar spine in asymptomatic subjects. A prospective investigation. J Bone Joint Surg Am 1990;72:403-8.

30. Okada E, Matsumoto M, Fujiwara H, Toyama Y. Disc degeneration of cervical spine on MRI in patients with lumbar disc herniation: comparison study with asymptomatic volunteers. Eur Spine J 2011;20:58591.

31. Medical Disability Advisor. Degeneration, lumbar intervertebral disc [Internet]. Westminster (CO): Reed Group Ltd.; c2013 [cited 2012 Aug 12]. Available from: http://www.mdguidelines.com/degenerationlumbar-intervertebral-disc.

32. Biluts H, Munie T, Abebe M. Review of lumbar disc diseases at Tikur Anbessa Hospital. Ethiop Med J 2012;50:57-65.

33. Thome C, Borm W, Meyer F. Degenerative lumbar spinal stenosis: current strategies in diagnosis and treatment. Dtsch Arztebl Int 2008;105:373-9.

34. Hochschuler SH. What you need to know about sciatica [Internet]. Deerfield (IL): Spine-health.com; c2013 [cited 2012 Aug 14]. Available from: http:// www.spine-health.com/conditions/sciatica/whatyou-need-know-about-sciatica.

35. Sirvanci M, Bhatia M, Ganiyusufoglu KA, et al. Degenerative lumbar spinal stenosis: correlation with Oswestry Disability Index and MR imaging. Eur Spine J 2008;17:679-85.

36. Ciricillo SF, Weinstein PR. Lumbar spinal stenosis. West J Med 1993;158:171-7.

37. Shambrook J, McNee P, Harris EC, et al. Clinical presentation of low back pain and association with risk factors according to findings on magnetic resonance imaging. Pain 2011;152:1659-65.

38. Beattie PF, Meyers SP, Stratford P, Millard RW, Hollenberg GM. Associations between patient report of symptoms and anatomic impairment visible on lumbar magnetic resonance imaging. Spine (Phila $\mathrm{Pa}$ 1976) 2000;25:819-28.

39. Genevay S, Atlas SJ. Lumbar spinal stenosis. Best Pract Res Clin Rheumatol 2010;24:253-65. 
40. Murphy DR, Hurwitz EL, Gregory AA, Clary R. A non-surgical approach to the management of lumbar spinal stenosis: a prospective observational cohort study. BMC Musculoskelet Disord 2006;7:16.

41. Altinkaya N, Yildirim T, Demir S, Alkan O, Sarica
FB. Factors associated with the thickness of the ligamentum flavum: is ligamentum flavum thickening due to hypertrophy or buckling? Spine (Phila Pa 1976) 2011;36:E1093-7. 